\title{
L. Condensation nuclei produced by cooling gases to low temperatures
}

\section{Gwilym Owen B.A. M.Sc. \& A.LI. Hughes B.Sc.}

To cite this article: Gwilym Owen B.A. M.Sc. \& A.LI. Hughes B.Sc. (1907) L. Condensation nuclei produced by cooling gases to low temperatures, Philosophical Magazine Series 6, 14:82, 528-538, DOI: $10.1080 / 14786440709463711$

To link to this article: http://dx.doi.org/10.1080/14786440709463711

曲 Published online: 16 Apr 2009.

Submit your article to this journal $[\pi$

Џ Article views: 2

Q View related articles 
(30) gives therefore

$$
\frac{d^{2} p_{2}}{d v^{2}}=\frac{\mathrm{RT}(v+b)}{a(v-b)^{3}}\left(\mu_{1}-\mu\right) .
$$

In the point $U$ just beneath the point $G, \mu_{1}-\mu>0$ and the $p_{2}-v$ curve turns therefore the convex side to the volume-axis. Because at the point $\mathrm{H}$ the tangent is parallel to the volume-axis, the $p_{2}-v$ curve has a point of flexion between $\mathrm{H}$ and $\mathrm{U}$. In the same manner we find that the $p_{2}-v$ curve HUWVK has also a point of flexion between $\mathrm{W}$ and $\mathrm{V}$, where $\mathrm{V}$ is the point just beneath $\mathrm{P}$.

The curve HUWVK gives therefore together with the empiric isotherm $\mathrm{HK}$ a complete image of the relation between the hydrostatic pressures $\left(p_{1}\right.$ and $\left.p_{2}\right)$ and the reciprocal value $v=\frac{1}{\rho}$ of the density in a point of the capillary layer, whereas the state of every point of the capillary is completely described by these three things.

I. Condensation Nuclei produced by Cooling Gases to Low Temperatures. By GWILYM OWEN, B.A., M.Sc., Assistant Lecturer and Demonstrator in Physics in the University of Liverpool, and A. LL. Hughes, B.Se.*

WE have recently examined the condition in which air is evolved from charcoal alter absorption in a dry and dust-free state at the temperature of liquid air, in order to determine whether it remains dust-free when liberated by warming the charcoal to atmospheric temperature. The charcoal was contained in the bend of a small glass U-tube connected to the cloud chamber of a Wilson's expansion apparatus; and, after the liquid air used for the cooling was removed and a sufficient time was allowed for the evolved air to regain the temperature of the atmosphere, a sample of the air was admitted through a glass tap into the cloud chamber. It was at once found that the evolved air contained large numbers of nuclei, sufficient, in fact, to produce dense showers ; but when a control experiment was performed without any charcoal in the U-tube, a similar number of nuclei appeared. The experiments therefore were indecisive as to whether the escape of the absorbed air caused disintegration of the charcoal, but they raised the very interesting question as to the cause of the showers obtained in the expansion chamber when air which had merely passed through a process of cooling

* Communicated by Prof. L. R. Wilberforce, M.A. 
produced by Cooling Gases to Low Temperatures.

was admitted. This peculiar effect we have investigated in a series of experiments extending over several months, and the present communication contains an account of the main results obtained hitherto.

It was at once found that it was not necessary to cool air to a temperature so low as that of liquid air in order to produce these condensation nuclei. Different gases bave been tried; and with all those experimented upon (except hydrogen) we have found that there is a more or less definite critical temperature below which each gas has to be cooled before the production of these nuclei can be detected. Further, the smaller the pressure of the gas while undergoing cooling, the lower is this eritical temperature. The investigation thus resolved itself into an attempt to determine these critical temperatures for various gases at different pressures, and thereby possibly to obtain some information regarding the origin of the nuclei.

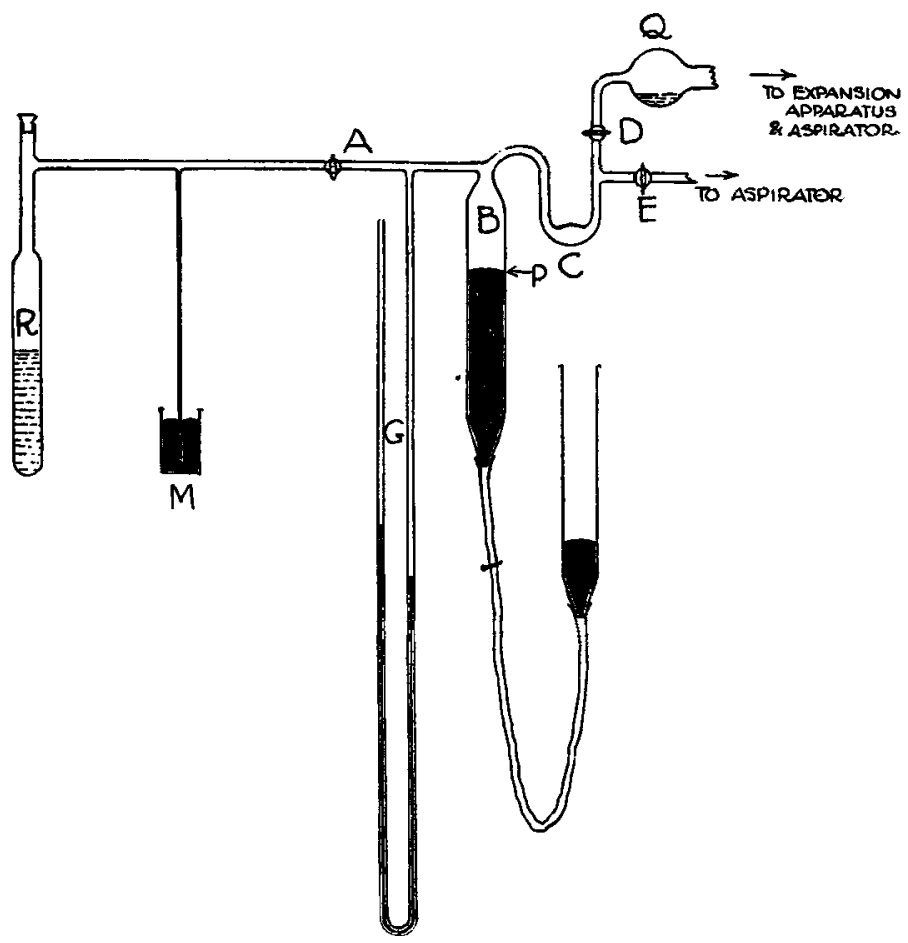

The final form of the apparatus used is represented in the figure. $Q$ is the cloud chamber of a Wilson's expansion 
apparatus * which, together with its accompanying gauge and mercury reservoir for adjusting the expansion, is omitted from the figure. The cooling was produced by immersing the bend of the glass tube $C$ always to the same depth in petroleum ether previously cooled by stirring with a test-tube containing liquid air. We find it convenient to give the name "tester" to the part of the apparatus immersed in the cooling liquid. The internal volume of the "tester" was about 3 cub. centims. The volume of the space ABCDE with the mercury in $B$ at a fixed mark $P$ was about 80 cub. cms. At the commencement of a test experiment this space was filled with the gas at a pressure of $80 \mathrm{cms}$. By raising or lowering the mercury in $\mathrm{B}$, the pressure of the gas in the "tester" could be increased to nearly two atmospheres or reduced to less than half an atmosphere. The gas was admitted through $\mathrm{A}$ and could be withdrawn through $\mathrm{E}$ or $\mathrm{D}$ by means of an aspirator. The tap $\mathbb{E}$ was found useful in rendering it possible to exhaust the apparatus to a low pressure in order to remove the gas lodging in the gauge $G$. Also by means of it a slow stream of gas would be drawn through the apparatus without there being any danger of water-vapour diffusing back into the "tester," as might conceivably happen when the gas was drawn through the cloud chamber via D. As a matter of fact, this fear proved to be groundless, the results obtained being independent of the path along which the gas was swept out of the "tester."

The method of making a test was as follows:-With the mercury at the top of the tube $B$, the gas was allowed to stream through the apparatus for several minutes in order to remove any nuclei produced by the previous test. The taps $\mathrm{D}$ and $\mathrm{E}$ were then shut and the mercury lowered in $\mathrm{B}$. By shutting $A$ off at a suitable moment, it could be so arranged that the pressure was $80 \mathrm{cms}$. when the mercury was at the fixed mark $\mathrm{P}$. In this way the same volume of gas was always experimented upon. The level of the mercury in $\mathrm{B}$ was now adjusted until the pressure was that proposed to use. One observer then surrounded the "tester" with the cooling liquid for 40 seconds, noting (by means of a pentane thermometer reading to $-200^{\circ}(\%)$ the temperature of the liquid at the beginning and end of the cooling process. The mean of these two readings was taken to be temperature which the gas in the "tester" attained. During the cooling the pressure of the gas was maintained constant by the second observer. The tester was now surrounded by water at a temperature of about $15^{\circ} \mathrm{C}$. for one and a half minutes. At

* C. T. R. Wilson, Phil. Trans. A. vol. clxxxix. p. 265 (1897). 
the end of this interval the mercury was again brought to the fixed mark P (so that the gas was once more at a pressure of 80 centims.). The tap D was now opened and the mercury quickly raised to a second fixed mark near the top of the tube, thereby driving into the cloud chamber practically the whole of the gas which had undergone the cooling. D was then closed and an expansion of about 1.10 made, completing the test. Between every test, whether nuclei had been produced or not, a stream of fresh gas was drawn through the apparatus for several minutes. At frequent intervals control tests were performed identical with that deseribed above omitting the cooling process only. Reasons are given below for selecting the stated duration of the cooling operation, the time allowed for the gas to warm up, and the value of the expansion used. It remains to add that always whenever the apparatus required resetting-up, it was first washed with caustic potash, nitric acid, and, after rinsing several times with clean water, thoroughly dried.

\section{General Results.}

The following facts in regard to the nuclei are general in that they apply to all the gases in which the nuclei were obtained.

(1) After passing below the "critical temperature" * the number of nuelei produced increases rapidly with the degree of cooling, the maximum effect being obtained when the cooling is sufficient to cause some of the gas to turn (or to be on the point of turning) into the liquid state. At this stage the number of nuclei produced is generally large enough to cause coloured clouds.

(2) The number of nuclei produced is independent of the time of cooling provided the time is long enough to allow the gas in the tester to fall to the temperature of the cooling liquid. This time was about 25 seconds with liquid air as the cooling agent. We allowed, however, a little margin, and in all the experiments described below the time of cooling was 40 seconds.

(3) The nuclei show a remarkable persistency. If an interval of 10 minutes be allowed to elapse between the removal of the cooling mixture and the passage of the gas into the cloud chamber, the shower obtained is almost as dense as it is when the warming-up process takes only one

* The term "critical temperature" in this paper means always that temperature to which a gas has to be cooled before the production of puclei can be detected. 
minute. When the number of nuclei produced is large, as is the case when the gas is cooled well below its "critical temperature," some nuclei can be detected quite half-an-hour after their formation. In all the experiments to be described we adopted a uniform interval of 90 seconds for the warmingup process, this interval being considered sufficiently long for the gas to acquire normal temperature and yet not long enough for an appreciable number of the nnclei to have disappeared.

(i) The number of nuclei "caught" is independent of the value of the expansion used. On using large expansions (greater even than an expansion of $1 \cdot 25$ which causes condensation on ions) no nuclei were detected in addition to the ordinary Wilson effect, where none had been detected with small expansions. On many occasions it was noticed that the nuclei caused condensation in consequence of the very slight supersaturation produced when adjusting the pressure-drop in the expansion apparatus preparatory to making an expansion. The small degree of supersaturation thus required to cause condensation on the nuclei proves them to be of considerable size. It is therefore unimportant what expansion is employed. We found it convenient to use a small expansion of $1 \cdot 10$ corresponding to a pressure-drop of 7 centims., and this was used throughout all the experiments described below.

\section{Determination of the "Critical Temperature."}

In contradistinction to the results given above, the following depend upon the nature and pressure of the gas cooled. The values of the "critical temperatures" given represent the mean estimates arrived at after a large number of experiments. The gases tried were ordinary air, air from boiling iquid air, hydrogen, oxygen, nitrogen, and carbon dioxide.

\section{Air from Boiling Liquid Air.}

Liquid air was poured through two thicknesses of filterpaper into the reservoir $\mathbf{R}$ (fig. 1 ) until the latter was balffull. The mouth of the reservoir was then closed by a small rubber stopper. When the tap $A$ was closed the air as it boiled away escaped through the mercury at M. The rate of boiling was regulated by surrounding $\mathrm{R}$ with a tall Dewar vessel also containing liquid air. Before commencing the experiment, the air escaping from $\mathbf{R}$ was allowed to stream for half an hour or longer through the apparatus escaping through $\mathrm{D}$ and $\mathrm{E}$ into the aspirator. While this was going 
on, the glass tube leading from $R$ to the "tester" and the tester itself were well heated with a flame so as to dry them thoroughly. The method of filling the apparatus with the gas preparatory to making a test has already been described.

The following table gives the "critical temperature" at different pressures for air obtained as described from boiling liquid air.

\begin{tabular}{|c|c|}
\hline $\begin{array}{c}\text { Pressure in centims. } \\
\text { of Mercury. }\end{array}$ & $\begin{array}{c}\text { "Critical Temperature" } \\
\text { (approximate). }\end{array}$ \\
\hline 101 & $-140^{\circ} \mathrm{C}$. \\
80 & $-145^{\circ} \mathrm{C.}$ \\
41 & $-160^{\circ} \mathrm{C}$. \\
\hline
\end{tabular}

Ordinary Air from outside.

The ordinary air was drawn slowly into the apparatus through long tubes containing calcium chloride, solid potash, phosphorus pentoxide, and cotton-wool.

The results obtained were practically identical with those tabulated above for air derived from liquid air.

\section{Hydrogen.}

The hydrogen was prepared by the action of $\mathrm{KOH}$ on pure aluminium. The gas passed from the generating vessel to the apparatus entirely through glass, no rubber connexions at all being used. On its way the gas was dried and purified by passing through sulphuric acid, calcium chloride, KOH, $\mathrm{P}_{2} \mathrm{O}_{5}$, and finally through a glass spiral about 2 feet long immersed in liquid air. A plug of glass-wool was inserted in the tube leading from the spiral to the main apparatus. No nuclei were ever detected in hydrogen until it had been cooled to $-175^{\circ} \mathrm{C}$. Generally, when hydrogen was cooled by means of liquid air, many nuclei were produced; but on one or two occasions the gas was obtained in such a state (possibly depending on the degree of purity) that no nuclei were formed at this low temperature, the pressure of the gas being approximately atmospheric. The "critical temperature" for hydrogen is therefore uncertain, but it is certainly very much lower than that obtained with all the other gases tried. 
534 Messi's. Owen and Hughes on Condensation Nuclei

\section{Oxygen.}

Oxygen was generated by heating a Jena-glass tube containing pure potassium permanganate. The gas passed through a long U-tube containing soda-lime, and then through a narrow glass tube 6 feet long bent into a spiral and immersed in liquid air. The oxygen was then condensed in a tube (similar to $\mathrm{R}$ in the figure) by surrounding it with liquid air. Enough oxygen was condensed to give about 20 litres of the gas. When this quantity of liquid oxygen was obtained the permanganate tube and spiral were cut off by means of a tap. The only piece of rubber used in the whole apparatus was the one connecting the permanganate tube to the tube of soda-lime.

The general results were almost identical with those obtained for air, the "critical temperature" at a pressure of 101 centims. being again about $-140^{\circ} \mathrm{C}$. At the lower pressure of 41 centims. the critical temperature seemed somewhat lower than in the case of air ; but on cooling by liquid air at this pressure, the oxygen in the tester liquefied and dense coloured showers or clouds were obtained.

\section{Nitrogen.}

Experiments were performed on nitrogen prepared in two ways. One method of preparation was to heat gently a mixture of equal parts of ammonium chloride, sodium nitrite, and potassium bichromate. The gas passed through $\mathrm{KOH}$, $\mathrm{H}_{2} \mathrm{SO}_{4}, \mathrm{CaCl}_{2}, \mathrm{P}_{2} \mathrm{O}_{5}$, the long spiral immersed in liquid air and a plug of glass-wool. The joints were all of sealed glass. This method is open to the objection that the nitrogen prepared in this way may contain traces of oxides of nitrogen.

The other method adopted was to drop ammonium chloride slowly into a solution of sodium hypobromite. The nitrogen evolved was then passed through sulphuric acid, over hot copper, through wash-bottles containing sulphuric acid, and potash, through calcium chloride and phosphorus pentoxide, the long spiral immersed in liquid air and a plug of cottonwool. The results for nitrogen are not so consistent as for air and oxygen, probably owing to its being so very difficult to obtain this gas in a pure state. At a pressure of 101 centims. the gas had only to be cooled to about $-125^{\circ} \mathrm{C}$. to canse the nuclei to appear. At the lower pressure of 41 centims., however, the "critical temperature" is approximately the same as in the case of air. 


\section{Carbon Dioxide.}

This gas was prepared from hydrochloric acid and marble and dried by passing through tubes of calcium chloride and phosphorus pentoxide. The results obtained with $\mathrm{CO}_{2}$ are illustrated by those given in the following table :-

\begin{tabular}{|c|c|c|c|}
\hline $\begin{array}{l}\text { Pressure in } \\
\text { centims. }\end{array}$ & $\begin{array}{l}\text { Temperature } \\
\text { of cooling. }\end{array}$ & $\begin{array}{l}\text { Effect obtained } \\
\text { in cloud chamber }\end{array}$ & Remarks. \\
\hline $\left.\begin{array}{c}35 \\
35 \\
\text { Pressure could not } \\
\text { be kept constant. }\end{array}\right\}$ & $\begin{array}{l}-73^{\circ} \mathrm{C} \\
-32 \\
-87\end{array}$ & $\begin{array}{l}5 \text { to } 10 \text { drops. } \\
\text { Thin shower. } \\
\text { Coloured cloud. }\end{array}$ & $\left\{\begin{array}{c}\text { Some of the gas } \\
\text { solidified. }\end{array}\right.$ \\
\hline $\left.\begin{array}{c}80 \\
80 \\
80 \\
\text { Pressure could not } \\
\text { be kept constant }\end{array}\right\}$ & $\begin{array}{l}-67 \\
-70 \\
-72 \\
-73\end{array}$ & $\begin{array}{l}\text { No effect. } \\
10 \text { to } 15 \text { drops. } \\
\text { Good shower. } \\
\text { Coloured cloud. }\end{array}$ & $\begin{array}{l}\text { Some gas solidified } \\
\text { Ditto. }\end{array}$ \\
\hline $\left.\begin{array}{l}117 \\
\text { Pressure could not } \\
\text { be kept constant. }\end{array}\right\}$ & $\begin{array}{l}-66 \\
-68\end{array}$ & $\begin{array}{l}5 \text { or } 6 \text { drops. } \\
\text { Very heavy rain. }\end{array}$ & Gas solidified. \\
\hline
\end{tabular}

We shall now consider certain possible explanations of the production of these nuclei and discuss their validity.

(1) By some direct action upon the walls of the cooled tube.This was disproved at the outset of the investigation by direct experiment, the gas in the tester being air. A double "tester" was made consisting of two similar glass U-tubes arranged side by side "in parallel" and symmetrically placed with respect to the tube $B$ and tap $D$ (see figure). One of the U-tubes contained a number of glass flakes obtained by breaking up a bulb of very thin glass. There was thus in this "tester" a much larger extent of glass surface, the volume of the air inside not being appreciably diminished. Cooling tests (by means of liquid air) were performed upon the "testers" alternately, and it was found that the effects obtained were practically the same ; if anything, the "tester" containing the flakes of glass gave a slightly smaller effect. This disproves the explanation that the effect is due to some action at the glass surface. There is also indirect evidence against this view. For, as we have shown, the effects depend in all cases upon the pressure and nature of the gas inside the "tester." Contrast for example the results for $\mathrm{CO}_{2}$ and Hydrogen. 
536 Messrs. Owen and Hughes on Condensation Nuclei

(2) Due to traces of water-vapour in the gas.-The importance of thoroughily drying the gas before admitting it into the experimental tube is obvious. Anv water-vapour present would be deposited on the cooled walls of the tube in the form of minute particles of ice. On the gas warming up again, these might become detached and float about in the gas in the form of small drops of water, and, consequently, if they did not evaporate (which, as a matter of fact, we might reasonably expect them to do, the gas in our experiments being very dry) they would act as condensation nuclei on admission into the cloud chamber.

Against this view that the nuclei are due primarily to traces of water-vapour in the gas, there are several pieces of evidence both direct and indirect.

(a) It has already been suggested that any drops of water formed as described above would evaporate on the gas warming up again. To help these supposed droplets to evaporate, on several occasions we surrounded the U-tube with hot glycerine at $120^{\circ} \mathrm{C}$. This treatment was ineffectual in removing them.

(b) All methods designed to dry the gas before its admission into the apparatus failed to prevent the formation of the nuclei at the temperatures already given. In the case of oxygen and air the gases were derived from boiling liquid oxygen and liquid air. The oxygen was dried before it was liquefied as has been described. The liquid air was poured into the reservoir through two layers of filter-paper in order to remove small particles of ice which necessarily fall into it from the mouth of the Dewar flask in which it was contained. Thus there could hardly hare been any appreciable amount of ice mixed with the liquid gases; and even if there were, its partial vapour-pressure at that temperature would be exceedingly small. (This applies equally to any traces of $\mathrm{CO}_{2}$ snow in the liquid gases.)

(c) The air from the room was on one occasion treated as follows :- It was drawn through calcium chloride, solid $\mathrm{KOH}$, over a long layer of $\mathrm{P}_{2} \mathrm{O}_{5}$, and finally through a spiral immersed in liquid air containing 17 feet of copper tubing of 1 millim. bore, the last foot being of wider bore packed with cotton-wool; yet after this drastic treatment the air bebaved no differently from what has been described above.

(d) Indirect evidence against the "moisture" explanation is afforded by the fact that the "critical temperature" at which the production of the nuclei can be first detected depends so markedly upon the nature of the gas. We might reasonably suppose all the gases tried to contain more 
produced by Cooling Gases to Low Temperatures.

or less the same amount of moisture (if any), and yet, as has been shown, the "critical temperatures" range from $-70^{\circ} \mathrm{C}$. to below $-175^{\circ} \mathrm{C}$.

The effects cannot therefore be due primarily to the gases experimented upon not being perfectly dry. Nor can the effects obtained in air and oxygen be due to traces of $\mathrm{CO}_{2}$, for we may again reasonably suppose the gases derived from boiling liquid air and liquid oxygen to be as free from $\mathrm{CO}_{2}$ as hydrogen prepared in the way described, yet the "critical temperature" for hydrogen is much lower than for air and oxygen. This view is strengthened by the fact that the "eritical temperature" for air mixed with nearly 4 per cent. of $\mathrm{CO}_{2}$ was raised only some 20 degrees.

(3) It might also be questioned if the gas had thoroughly warmed up before admission into the cloud chamber, for the entrance of cold gas into the moist gas in the latter might conceivably of itself produce nuclei. To invalidate this contention, it is only necessary to point out that hydrogen cooled to $-150^{\circ} \mathrm{C}$. say, would not have had as good a chance to regain atmospheric temperature in the 90 seconds interval allowed as $\mathrm{CO}_{2}$ cooled to $-80^{\circ} \mathrm{C}$.; yet no effect is produced in the former, and dense elouds in the latter case. The fact that showers can be obtained on admitting the gas into the cloud chamber ten minutes after the cooling agent is removed still further disarms this criticism.

\section{Discussion of the Results.}

We are by no means sure as to the correct explanation of the origin of the nuclei produced in a gas, as we have described, by severe cooling. As a possible explanation of the effects obtained in bydrogen, air, oxygen, and nitrogen, we tentatively propose the view that when gases are cooled to a sulficiently low temperature, yet considerably higher than their real liquefying points, the molecules of the gas come together and form aggregations of a considerable size which in some way or other (at present very difficult to understand) are able to persist for a long time after the gas has regained its normal temperature. In other words, the effects suggest the interesting fact that what we might call incipient liquefaction occurs in these gases at temperatures well removed from their real liquefying points. It may also be pointed out that, broadly speaking, the lower the real liquefying point of a gas, the lower is its "critical temperature," and the greater is the temperature-distance between the "critical temperature" and the real liquefying point. The results for nitrogen at a 
high pressure show a departure from this rule, possibly owing to unavoidable impurities.

In the case of $\mathrm{CO}_{2}$ it will be noticed that the effect obtained is much more marked when some of the gas is actually solidified during the cooling process. It might therefore be urged that with $\mathrm{CO}_{2}$ the nuclei are produced not while the gas is approaching the solid state, but while changing from the solid back again into the gaseous condition. This view, however, is improbable on the analogy of the results obtained with the other gases. It is further disqualified by a fact which we have not yet mentioned, namely, that the air derived fro: $n$ boiling liquid air is perfectly nuclei-free, so that (as may be seen from the figure) no glass or cotton-wool plug had to be inserted between the reservoir containing the liquid air and the experimental tube. This fact indeed confirms the ordinary view of evaporation, that it is due to the escape of separate molecules from the free surface of the liquid. Evidently then in the case of air the formation of these nuclei is not a "reversible" process. This point was not tested in the case of liquid oxygen as there happened to be a glasswool plug already in position, but there is no reason for supposing that oxygen would behave differently in this respect from liquid air. The point raised in connexion with the effects in $\mathrm{CO}_{2}$ is at present left open ; we hope to investigate it later on among other aspects of the problem.

In conclusion we have pleasure in acknowledging our indebtedness to Prof. L. R. Wilberforce for numerous suggestions and for the facilities he so readily placed at our disposal. Our best thanks are also tendered to Professor F. G. Donnan for very helpful advice in regard to the preparation of some of the gases.

George Holt Physics Laboratory,

The University of Liverpool, August 1, 1907.

LI. On the Electromagnetic Mass of a Moving Electron. By E. Cunningham, St. John's College, Cambridge*.

$\mathbf{I}^{\mathrm{T}}$

$\mathrm{N}$ his discussion of the electromagnetic mass of a moving electron (Theorie der Elektrizität, ii. p. 205), Abraham raises an objection to the Lorentz conception of an electron as having, at rest, a spherical shape, but in motion the shape of an oblate spheroid the ratio of whose axes is $\sqrt{1-v^{2} / c^{2}}$, $v$ being the velocity relative to the rother, and $c$ the velocity

* Communicated by the Author. 